\title{
Tests of the lattice index theorem
}

\author{
Gerald Jordan. * Roman Höllwieser, and Manfried Faber \\ Atomic Institute, Vienna University of Technology, \\ Wiedner Hauptstr. 8-10, 1040 Vienna, Austrid \\ Urs M. Heller \\ American Physical Society, One Research Road, Box 9000, Ridge, NY 11961-9000, USA
}

(Dated: October 31, 2018)

\begin{abstract}
We investigate the lattice index theorem and the localization of the zero-modes for thick classical center vortices. For non-orientable spherical vortices, the index of the overlap Dirac operator differs from the topological charge although the traces of the plaquettes deviate only by a maximum of $1.5 \%$ from trivial plaquettes. This may be related to the fact that even in Landau gauge some links of these configuration are close to the non-trivial center elements.
\end{abstract}

PACS numbers: 11.15.Ha, 12.38.Gc

\section{INTRODUCTION}

At large separations static color sources of non-vanishing N-ality give rise to gluonic flux tubes and a linearly rising potential. This behavior is caused by the non-perturbative QCD-vacuum which compresses the gluonic flux. Center vortices [1, 2, 3, 4, 5, 6], quantized magnetic flux lines, play an important role for the confinement of color charges, as has been shown by numerical calculations [7, 8]. In addition, simulations have indicated that vortices could also account for phenomena related to chiral symmetry, such as topological charge and spontaneous chiral symmetry breaking (SCSB) $9,10,11,12$.

It is well-known that these non-perturbative features of QCD are intimately linked to the properties of the low-lying spectrum of the Dirac operator. The topological charge of a gauge field equals the index of the Dirac operator, while the chiral condensate, the order parameter for SCSB, is proportional to the spectral density of the near-zero modes.

With the advent of the overlap operator 13, 14], the fundamental problems of investigating chiral features on the lattice have been overcome. The overlap operator has an exact chiral symmetry [15], implements a lattice version of the index theorem [13], and may even be used for the definition of a local topological charge density [9].

In this paper, we report on our calculations with the overlap operator applied to thick classical center vortices. We investigate the localization of zero-modes with respect to the position of the thick vortices and find an interesting discrepancy in the topological charge determined by different methods.

\section{STRATEGY}

We work with $\mathrm{SU}(2)$ gauge fields on lattices with $N_{s}$ sites in the x-, y- and z-direction, and $N_{t}$ sites in the t-direction. In four dimensions vortices form closed two-dimensional surfaces. We investigate thick classical center vortices in the shapes of planes (closed by lattice periodicity) and spheres. The details of the individual vortex types will be discussed along with our results.

We compare different definitions of the lattice topological charge:

(1) The topological charge determined from the shape of P-vortices, that means from vortex intersections and writhing points according to ref. 9 ].

(2) The index of the overlap Dirac operator 13, 14].

According to the Atiyah-Singer index theorem, which holds for continuous fields, the topological charge is given by the index

$$
\operatorname{ind} D[A]=n_{-}-n_{+}=Q
$$

\footnotetext{
*Also at Photonics, Vienna University of Technology, Gusshausstrasse 25-29, 1040 Vienna, Austria.

${ }^{\dagger}$ Electronic address: faber@kph.tuwien.ac.at
} 
where $n_{-}$and $n_{+}$are the number of left- and right-handed zero modes [16, 17, 18]. It has been shown that this relation is also valid on the lattice for the overlap Dirac operator: its index coincides with the discretized gluonic definition in the continuum limit [19].

The overlap Dirac operator is defined by [20]

$$
D=\frac{1}{2}\left[1+\gamma_{5} \epsilon\left(H_{L}^{+}\right)\right]
$$

Here, $\epsilon$ is the sign function,

$$
H_{L}^{+}=\gamma_{5} D_{w}\left(-m_{0}\right)
$$

and $D_{w}$ is the usual lattice Wilson Dirac operator with mass $-m_{0}$ (we use $m_{0}=1.0$ ).

The overlap operator can be used to define the topological charge only on so-called "admissible" gauge fields. This restriction assures that $H_{L}^{+}$has no zero eigenvalues so that the sign-function is well-defined. A sufficient, but not necessary condition for this is [21, 22]

$$
\operatorname{tr}\left(\mathbb{1}-U_{\mu \nu}\right)<0.03
$$

The diagonalization of the overlap operator yields the corresponding Dirac zero modes with well-defined chirality properties. We further look at the distribution of the scalar fermionic density

$$
\rho(x)=\sum_{c, d}\left|\vec{v}(x)_{c d}\right|^{2},
$$

where the summation indices $c$ and $d$ refer to color and Dirac indices of the eigenvectors $\vec{v}$, respectively, and the left- or right-handed chiral densities

$$
\rho_{ \pm}(x)=\sum_{c, d, d^{\prime}} \vec{v}(x)_{c d}^{*} \frac{\left(1-\gamma_{5}\right)^{d, d^{\prime}}}{2} \vec{v}(x)_{c d^{\prime}}^{2}
$$

where only zero-modes of the corresponding chirality contribute. When the zero modes of a specific chirality are degenerate, it is useful to investigate the square root of the sum of the scalar densities of these modes. This average density respects eventual symmetries of the field configuration.

(3) The integral (sum, on the lattice) of the gluonic charge density $q(x)=\frac{1}{16 \pi^{2}} \operatorname{tr}\left(\mathcal{F}_{\mu \nu} \tilde{\mathcal{F}}_{\mu \nu}\right)$ in the "plaquette" and/or "hypercube" definitions on the lattice, see ref. [23, 24]. Since Monte-Carlo configurations are in general too coarse, these definitions are usually only applied after cooling.

In the continuum, since the topological charge density $q(x)$ is the total derivative of the topological current $k_{\mu}$ [25],

$$
\begin{aligned}
q(x) & =\partial_{\mu} k_{\mu} \\
k_{\mu} & =-\frac{1}{8 \pi^{2}} \epsilon_{\mu \nu \rho \sigma} \operatorname{tr}\left[\mathcal{A}_{\nu} \partial_{\rho} \mathcal{A}_{\sigma}+\mathrm{i} \frac{2}{3} \mathcal{A}_{\nu} \mathcal{A}_{\rho} \mathcal{A}_{\sigma}\right]
\end{aligned}
$$

one can apply Gauss' theorem to transform the expression for $Q$ into a surface integral,

$$
Q=\int d^{4} x q(x)=\oint_{S^{3}} d \sigma_{\mu} k_{\mu}
$$

provided the gauge field is smooth. Assuming a sufficiently fast decaying field strength for $x^{2} \rightarrow \infty$, the gauge field at infinity can be written as a pure gauge

$$
\mathcal{A}_{\mu}\left(x^{2} \rightarrow \infty\right)=\mathrm{i} \partial_{\mu} \Omega \Omega^{\dagger}
$$

It can be shown that the surface integral Eq. (8), and hence $Q$ for smooth fields, measures the winding number of $\Omega$, which maps the group space $\mathrm{SU}(2)$ onto the space-time surface $S^{3}$ at infinity. 

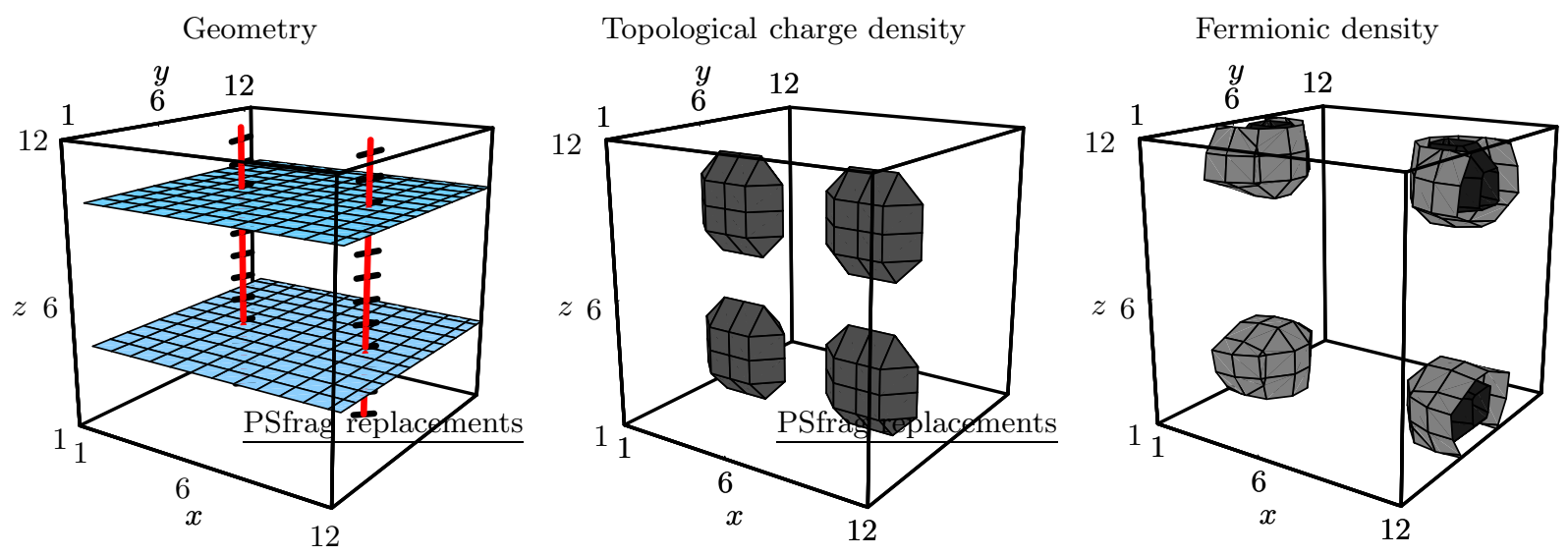

FIG. 1: Plane vortices on a $12^{4}$-lattice in xy- and zt-plane intersect in four points giving topological charge and fermionic density.

\section{DESCRIPTION OF CONFIGURATIONS AND RESULTS}

\section{A. Plane vortices}

The construction of planar vortices is explained in [26]. We use periodic boundary conditions where plane center vortices always come in pairs. All three definitions of $Q$ yield identical results for all configurations containing only plane vortices.

As an example, we show in Fig. 1 some diagrams for two orthogonal pairs of plane vortices on a $12^{4}$ lattice, which intersect in 4 places. The left picture shows the position of the P-plaquettes after going to maximal center gauge and center projection. One vortex pair extends in $x-y$-planes, the other pair in z-t-planes. The short pieces of lines attached to the vertical line symbolize the extent of the $z$ - $t$-vortices in the time-direction. The diagram in the middle of Fig. 1 shows an equi-density surface of the topological charge density determined in the plaquette definition and the right diagram shows an equi-density surface of the scalar fermionic density.

Each of the four intersection points gives rise to a lump of topological charge $Q= \pm \frac{1}{2}[9]$. The sign of the contribution at a given intersection point can be changed by a flip of the orientation of the vortex surface, this means by a transition from links $U_{\mu}(x)$ creating one of the vortices to $U_{\mu}^{\dagger}(x)$ in the region of the intersection. After abelian projection a smooth transition of the vortex orientation in color space produces a monopole line. In this way one can produce configurations with two vortex pairs of topological charge $Q=0, \pm 1$ or \pm 2 . In all these cases the lattice index theorem is fulfilled and our results agree with the analytical solution for the zero modes presented in [27].

\section{B. A non-orientable spherical vortex}

A non-orientable spherical vortex can be constructed with an appropriate "profile"-function $\alpha\left(\left|\vec{r}-\vec{r}_{0}\right|\right)$ by time-like links which form a hedgehog in one time-slice of the lattice, where $\vec{r}_{0}$ is the center of a spatial sphere

$$
U_{\mu}(x)= \begin{cases}\exp \left\{\mathrm{i} \alpha\left(\left|\vec{r}-\vec{r}_{0}\right|\right) \vec{n}(x) \cdot \vec{\sigma}\right\} & t=1, \mu=4 \\ \mathbb{1} & \text { elsewhere }\end{cases}
$$

A characteristic property of a hedgehog configuration is the agreement between the color direction $\vec{n}$ and the spatial direction of $\vec{r}-\vec{r}_{0}$

$$
\vec{n}(\vec{r}, t)=\frac{\vec{r}-\vec{r}_{0}}{\left|\vec{r}-\vec{r}_{0}\right|}
$$

see left diagram in Fig. 2. For the choice (10) of the links the time-like Wilson lines (holonomies) agree with the time-like links in the $t=1$ time-slice.

At $\vec{r}=\vec{r}_{0}$ and at the largest possible distances from $\vec{r}_{0}$ on a periodic lattice the direction of the color-vectors (11) are undefined. This does not lead to a singularity of the gauge field if the profile-function $\alpha(r)$ is appropriately 

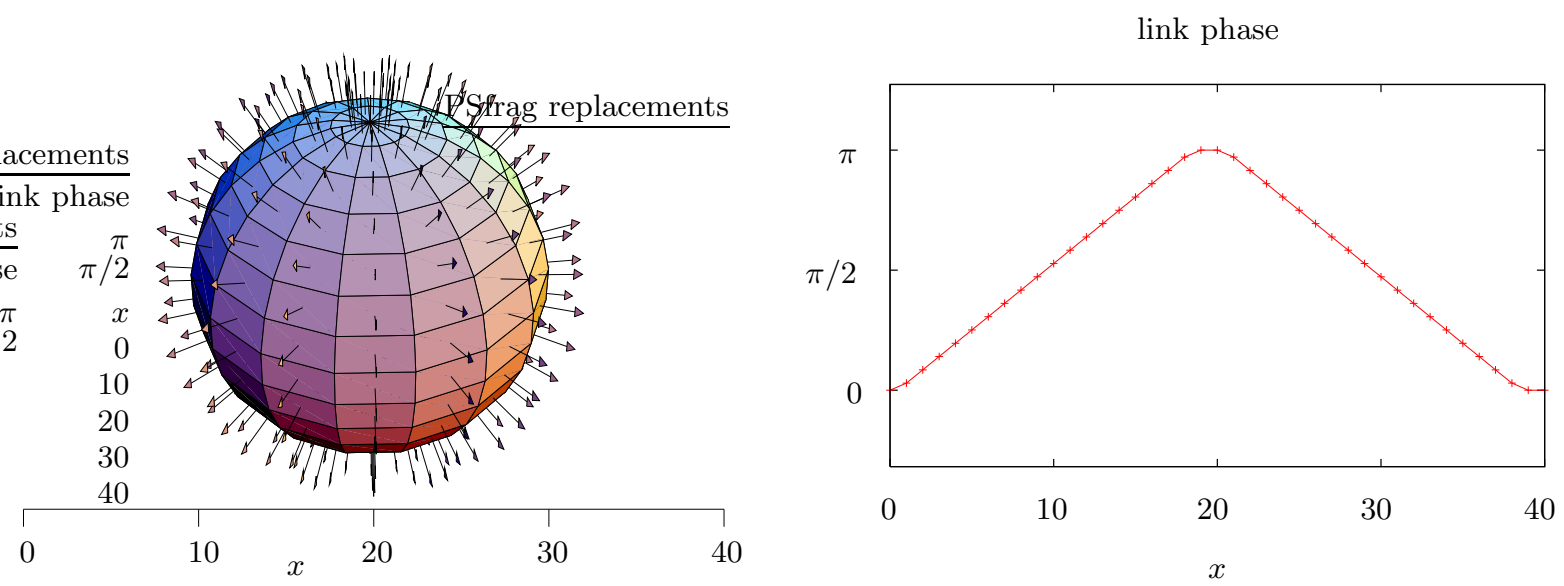

FIG. 2: Thick Spherical SU(2)-vortex (hedgehog, non-orientable) and change of its link phase $\alpha(r)=\alpha_{-}(r)$ according to Eq. (10).

chosen such that the Wilson lines at these special points become center elements of the gauge group. A good choice is $\alpha(r)=\alpha_{-}(r)$ with

$$
\alpha_{-}(r)= \begin{cases}\pi & r<R-\frac{\Delta}{2} \\ \frac{\pi}{2}\left(1-\frac{r-R}{\frac{\Delta}{2}}\right) & R-\frac{\Delta}{2}<r<R+\frac{\Delta}{2} \\ 0 & R+\frac{\Delta}{2}<r .\end{cases}
$$

As shown in the right diagram of Fig. 2 for a $40^{3} \times 2$ lattice the phase $\alpha$ of the links in the first time slice varies from $\pi$ at the center of the sphere to 0 outside of the vortex core of thickness $\Delta$, chosen as $\Delta=18$.

The check that this configuration is a vortex is done with maximal center gauge and center projection and results in a P-vortex forming a lattice representation of a sphere of radius 10 in the first time slice. The color structure of the thick vortex which is symbolically indicated in the left diagram of Fig. 3 leads to a monopole loop on a great circle of the P-vortex after maximal abelian gauge and abelian projection. The direction of the loop depends on the $\mathrm{U}(1)$ subgroup chosen as abelian degrees of freedom. For the subgroup defined by the Pauli matrices $\sigma_{1}, \sigma_{2}$ or $\sigma_{3}$ the monopole loops are in the $\mathrm{y}-\mathrm{z}-, \mathrm{z}-\mathrm{x}-$ and $\mathrm{x}-\mathrm{y}$-plane, respectively. This is indicated schematically in Fig. 3 with three colors.
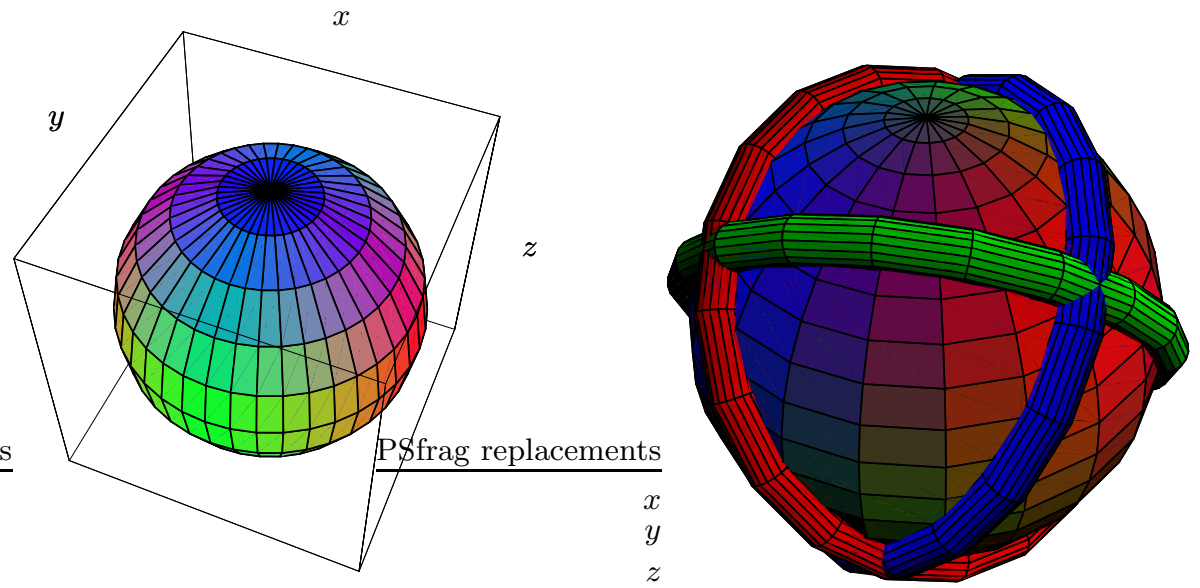

FIG. 3: The color structure of the vortex surface (1) leads to monopole lines after abelian projection (r).

The P-vortex surface which is a closed surface in dual space consists only of the duals of space-time plaquettes. There are no vortex intersections and no writhing points. Therefore, the topological charge $Q$ determined from the P-vortex surface [9] vanishes, $Q=0$. The traces of all plaquettes are close to unity, $\operatorname{tr}\left(\mathbb{1}-U_{\mu \nu}\right) \leq 1-\cos \frac{\pi}{18}=0.015$, 

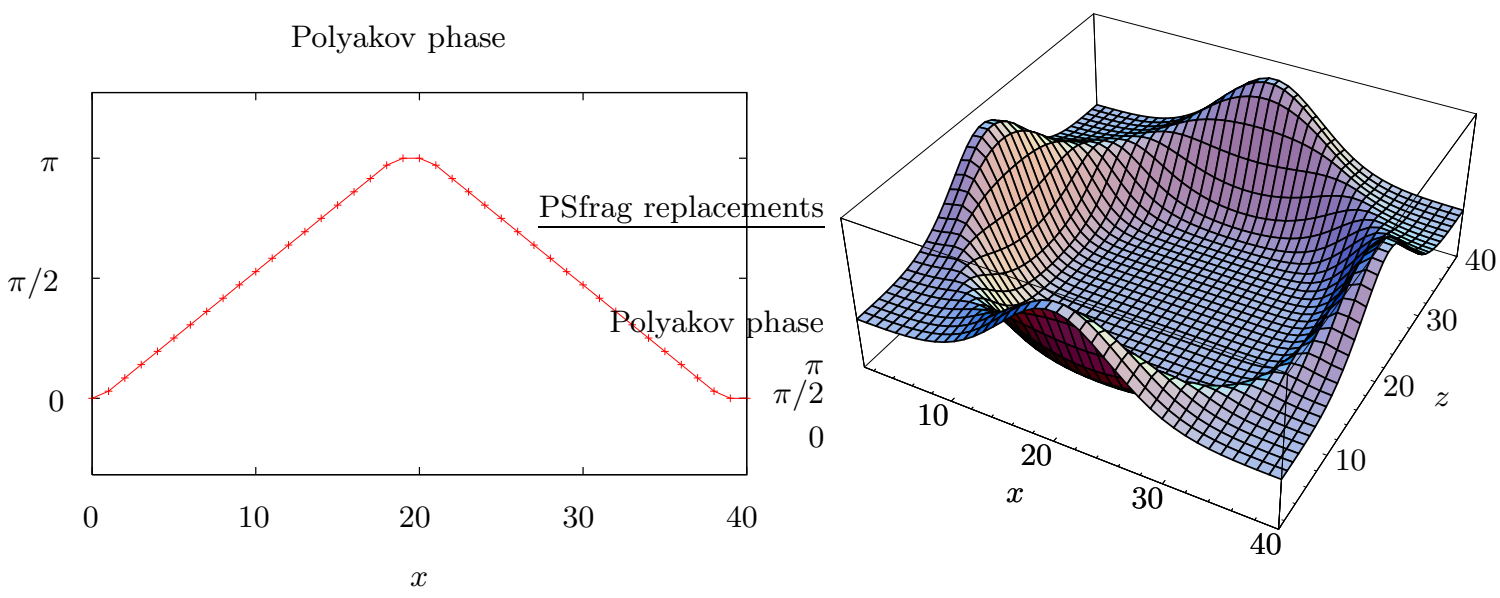

FIG. 4: Phases of Wilson lines along a line through the center of a spherical vortex (1), normalized scalar densities for all zero-modes of one chirality in the planes with $z=20$ close to the center of the sphere (r). The density vanishes in the region where the phase of the Polyakov loop is $\pi$. Both chiralities and both time-slices on the $40^{3} \times 2$-lattice give the same picture.

compare the link-phases in Fig. 2. The topological charge in the plaquette definition or in the hypercube definition can be used safely without cooling. According to Eq. (10) the only non-trivial links are time-like, therefore all space-space plaquettes are trivial, which confirms the vanishing of the topological charge.

This configuration has three zero-modes [29] of positive chirality and four zero-modes of negative chirality. Normalized densities of positive and negative chirality modes have the same spatial distribution.

For the spherical vortex with center at $(19.5,19.5,19.5)$, we show the scalar density in the plane $z=20$ in Fig. 4. The non-trivial links connect $z=20$ and $z=21$, and the densities in both time-slices are identical. The zero-modes are localized mainly in the region of trivial Wilson lines (for $\left|\vec{r}-\vec{r}_{0}\right| \sim 20$ ) and seem compressed in the regions where the mirror pictures of the spherical vortex on the periodic lattice approach each other. In the region where the Wilson lines are non-trivial center elements (for $\left|\vec{r}-\vec{r}_{0}\right|$ around 0 ), the density vanishes. According to the index theorem the topological charge is given by

$$
Q=n_{-}-n_{+}=1
$$

This value is in disagreement with the values determined above $(Q=0)$. But it agrees with the gluonic result which one gets after cooling. The cooling history is shown in Fig. 5. The left scale shows the action $S=\sum_{\square}\left(1-\frac{1}{2} \operatorname{Tr} U_{\square}\right)$ in units of the one-instanton action $S_{\text {inst }}$ and the right scale the value of the topological charge in the plaquette definition. The spherical vortex is not a minimum of the action, i.e. not an instanton. During cooling its action is first rapidly decreasing, whereas its topological charge starts with zero and reaches a value close to one after a few cooling steps. On the $40^{3} \times 2$-lattice the action does not really approach a plateau value. Since a plateau appears for a $12^{4}$-lattice this seems to be related to the strong asymmetry of the lattice. Hence one would expect to see a clear plateau on a symmetric, but finer $40^{4}$ lattice, as well. The number of zero-modes is the same for various lattice sizes we used: $12^{4}$, and $12^{3} \times 2$ up to $40^{3} \times 2$, increasing the spacial extent $L_{s}$ in steps of 4 . We conclude that cooling and overlap fermions give a different topological charge than the discretized integral $\frac{1}{16 \pi^{2}} \int d^{4} x \operatorname{tr}\left(\mathcal{F}_{\mu \nu} \tilde{\mathcal{F}}_{\mu \nu}\right)$ even for very smooth field configurations.

The hedgehog configuration is characterized by links in the center of the spatial sphere which are non-trivial center elements and Wilson lines $L(\vec{x}), \vec{x}=(x, y, z)$, with a topological winding number $\nu=1$,

$$
\nu(\{L\})=\frac{1}{24 \pi^{2}} \int \mathrm{d}^{3} r \epsilon_{i j k} \operatorname{Tr}\left(V_{i} V_{j} V_{k}\right), \quad V_{i}=\partial_{i} L L^{\dagger} .
$$

The topological charge that we get in the cooling process and from the index theorem agrees with this winding number. In the continuum limit, the gauge field at the center of the hedgehog becomes singular. This singularity is due to the topological obstruction and can't be removed by a gauge transformation. With Landau-gauge, which we did for 100 gauge copies on a $40^{3} \times 2$-lattice, it is not possible to get rid of links close to non-trivial center elements. For the lattice spacing $a$ approaching zero, the gauge field for these links, $\mathcal{A}_{0}(x) \approx \pi \sigma_{3} /(g a)$, diverges. In all gauge copies we found the same links with negative traces. The number of such negative links and the values of their traces are listed in Table \ 


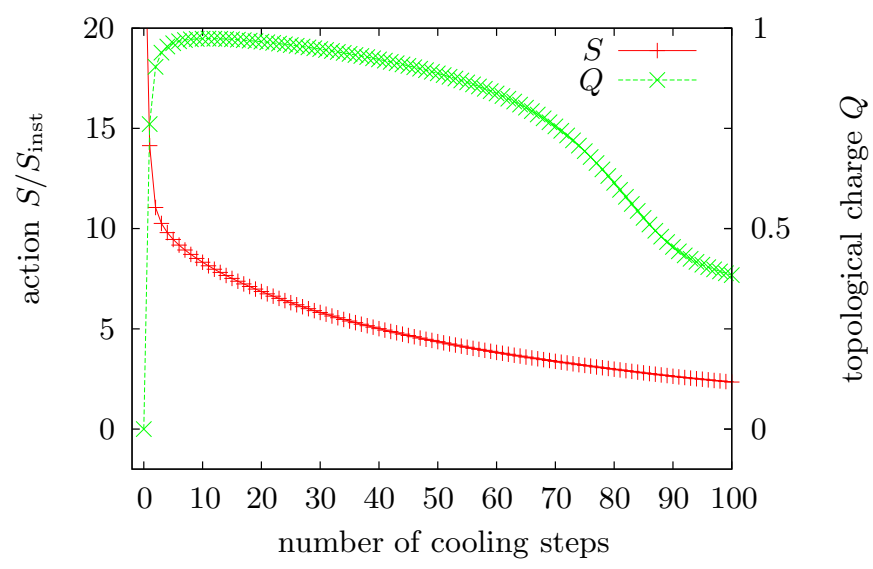

FIG. 5: By cooling the topological charge rises from zero close to one for $\alpha=\alpha_{-}$(right scale) while the action $S$ (in units of the one-instanton action $S_{\text {inst }}$ ) decreases slowly (left scale). The data is for a $40^{3} \times 2$-lattice.

\begin{tabular}{|c|ccccccc|}
\hline number of links & 8 & 24 & 24 & 32 & 48 & 24 & 48 \\
\hline$\frac{1}{2} \operatorname{tr} U_{\mu}(x)$ & -0.93 & -0.68 & -0.46 & -0.30 & -0.18 & -0.08 & -0.01 \\
\hline
\end{tabular}

TABLE I: Number of links with negative traces and trace values after Landau gauge for the non-orientable spherical vortex of Eq. (10) on a $40^{3} \times 2$-lattice, e.g. there are eight links with $\frac{1}{2} \operatorname{tr} U_{\mu}(x)=-0.93$.

\section{Other configurations with spherical vortices}

An orientable spherical vortex we construct with the prescription

$$
U_{\mu}(x)= \begin{cases}\exp \left\{\mathrm{i} \alpha\left(\left|\vec{r}-\vec{r}_{0}\right|\right)\left|n_{i}(x)\right| \cdot \sigma_{i}\right\} & t=1, \mu=4, \\ \mathbb{1} & \text { elsewhere }\end{cases}
$$

Here, $\left|n_{i}(\vec{r}, t)\right|=\left|\frac{\left.\vec{r}-\vec{r}_{0}\right)_{i}}{\left|\vec{r}-\vec{r}_{0}\right|}\right|$ is the absolute value of the coordinates of the direction vector $\vec{n}$. These links cover only half of the SU(2) group space and this half they cover twice. The time-like Wilson lines have topological winding number zero. This configuration has no zero-modes and all ways to determine the topological charge lead to the same result, $Q=0$. With maximal Abelian gauge no monopole lines appear. With Landau-gauge, which we did for 100 gauge copies on a $40^{3} \times 2$-lattice, one can get rid of links close to non-trivial center elements. The number of such negative links and the values of their traces are listed in Table 1 .

$$
\begin{array}{|c|cccc|}
\hline \text { number of links } & 8 & 24 & 24 & 24 \\
\hline \frac{1}{2} \operatorname{tr} U_{\mu}(x) & -0.26 & -0.15 & -0.06 & -0.008 \\
\hline
\end{array}
$$

TABLE II: Number of links with negative traces and trace values after Landau gauge for the orientable spherical vortex of Eq. (15) on a $40^{3} \times 2$-lattice.

A second type of a non-orientable spherical vortex we construct with the prescription (10) and the profile function $\alpha(r)=\alpha_{+}(r)$,

$$
\alpha_{+}(r)= \begin{cases}0 & r<R-\frac{\Delta}{2} \\ \frac{\pi}{2}\left(1+\frac{r-R}{\frac{\Delta}{2}}\right) & R-\frac{\Delta}{2}<r<R+\frac{\Delta}{2} \\ \pi & R+\frac{\Delta}{2}<r\end{cases}
$$

The phases of the links in the time slice agree again with the phases of the Wilson lines in time direction and are shown in Fig. 6. In the center of the spherical vortex the temporal Wilson lines are trivial and at "infinity" they are 
non-trivial center elements. This configuration gives only one zero-mode of negative chirality and none of positive chirality. The zero-mode is localized in the center of the spatial sphere and in both time-slices occupied by the vortex. Again, the scalar density prefers regions of trivial Wilson lines over those of non-trivial values. According to the index theorem the topological charge is -1 and agrees with the winding number (14) of the hedgehog of Wilson lines. The topological charge computed from plaquettes is $Q=0$ while the plaquette traces differ by less than $1.5 \%$ from trivial ones. Since this configuration is related to the configuration in Fig. 4 by a non-periodic gauge transformation, it is not surprising that again the different determinations of the topological charge do not agree.
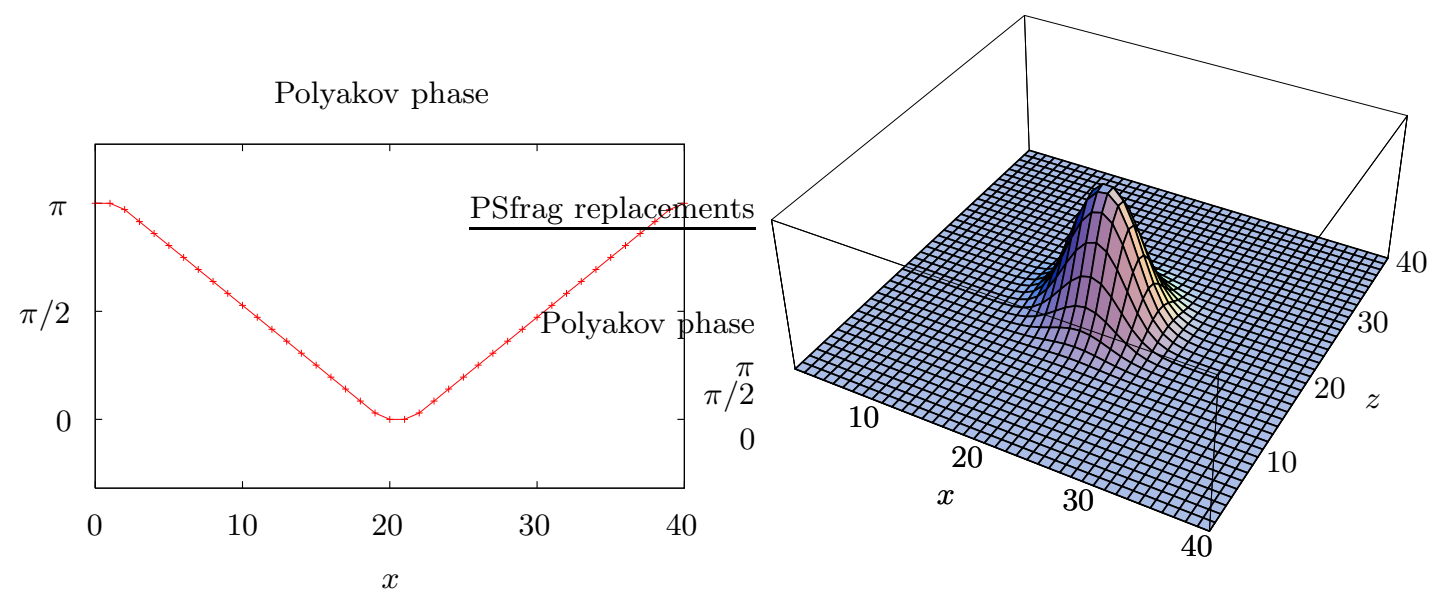

FIG. 6: Phase-diagram of the Polyakov loop (l) along a line through the center of a spherical vortex with the profile function $\alpha=\alpha_{+}$(l) and scalar density of the only fermionic zero mode (r) which has positive chirality.

For two equal vortices of this type, $\alpha=\alpha_{+}$, one in time slice $t=1$ and another at $t=2$ of the $40^{3} \times 2$-lattice we get three zero-modes of positive chirality with a scalar density like the diagram in Fig. 4 and five of negative chirality with the densities shown in Fig. 7 .

Since the modes are degenerate, they can mix and the plots display the sum of the densities of all zero-modes. The left diagram displays the $t=1$ slice, where only four of these modes contribute significantly, while the fifth mode leads only to the small peak in the middle. Conversely, the density in the $t=2$ slice (shown in the right picture) is dominated by the fifth mode in the center, the other modes being of a magnitude invisible on this scale.

The phase of the Polyakov line for this configuration is zero in the center of the spherical vortices and due to the $2 \pi$-periodicity in the region between the mirror pictures on the periodic lattice. Again the zero-modes are localized in the region with trivial time-like Wilson lines.

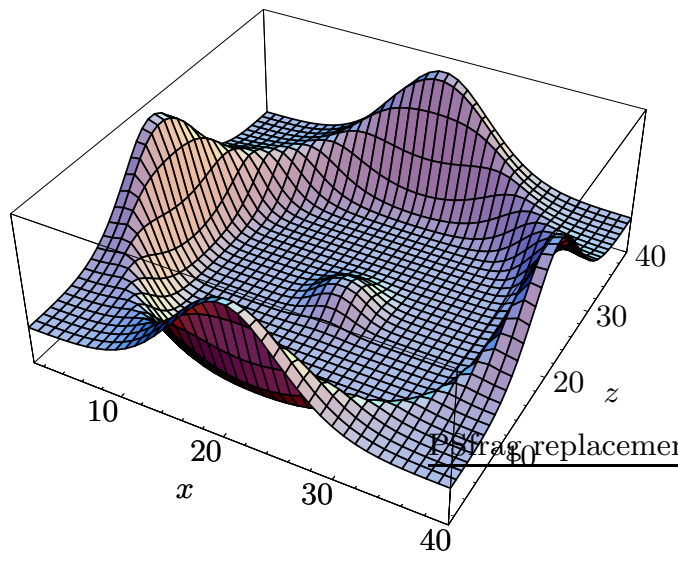

$t=1$

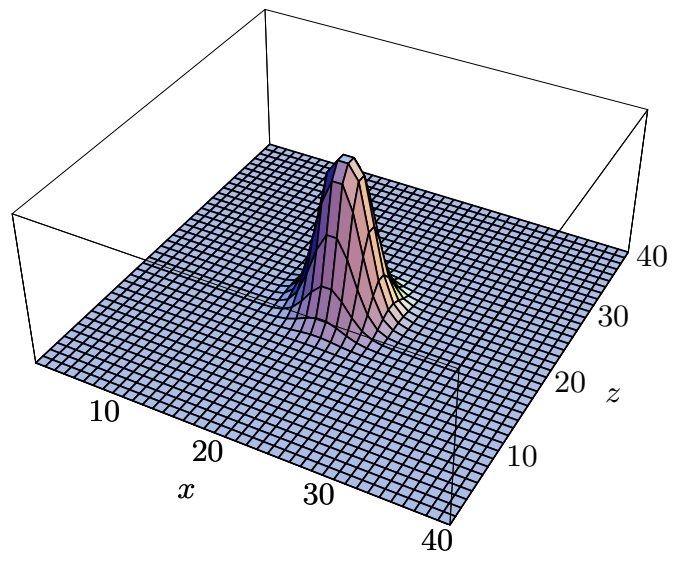

$t=2$

FIG. 7: Scalar densities of fermionic zero modes of negative chirality for two equal vortices with the profile function $\alpha=\alpha_{+}$, in the time slices $t=1$ and $t=2$ of the $40^{3} \times 2$-lattice. 
A configuration with two vortices of different type in the two time-slices, one with $\alpha=\alpha_{-}$at $t=1$ and another with $\alpha=\alpha_{+}$at $t=2$ does not show any zero-mode. In this case there is no discrepancy in the determinations of the topological charge. The phase of the Wilson line, which is the sum of the phases from the two vortices, is constant and equal to $\pi$.

A summary of the the number of zero-modes for the configurations described above with spherical vortices is given in Table III

\begin{tabular}{|c|cc|}
\hline profile $\alpha$ & $n_{+}$ & $n_{-}$ \\
\hline$\alpha_{-}$at $t=1$ & 3 & 4 \\
$\alpha_{+}$at $t=1$ & 1 & 0 \\
$\alpha_{-}$at $t=1$ and $\alpha_{+}$at $t=2$ & 3 & 5 \\
$\alpha_{+}$at $t=1$ and $\alpha_{-}$at $t=2$ & 5 & 3 \\
$\alpha_{+}$at $t=1$ and $\alpha_{+}$at $t=2$ & 0 & 0 \\
\hline
\end{tabular}

TABLE III: Number of zero-modes for various smooth field configurations with spherical vortices. The profile functions $\alpha_{ \pm}$are defined in Eq. (12) and (16).

The above table can be summarized in the following empirical rule: Spherical vortices in slices (3D volumes) of the lattice contribute to the Dirac operator index with an integer given by the winding number (14) of the corresponding Wilson lines, mapping the 3D volume of the slice to the $\mathrm{SU}(2)$ manifold of the Wilson lines.

\section{CONCLUSION}

We have investigated various configurations with plaquettes close to unity. For configurations with a non-zero winding number of the Wilson lines we get disagreement between the topological charge determined by the plaquette and hypercube definition, and from the vortex intersections and writhing points on one side, and the topological charge from the index theorem and cooling on the other side. The transition in Eq. (8), from the 4D integral over the topological charge density, to the 3D integral of the $\mathrm{SU}(2)$-valued map $\Omega$, is only possible for smooth gauge fields. Vortex configurations with non-trivial winding numbers have singularities in the gauge fields which can't be removed by gauge transformations or smooth deformations of the field configuration. According to the vortex model of confinement vortices form closed two-dimensional surfaces which in the confined phase penetrate the whole space-time. In ref. 28. it was shown that P-vortex surfaces are mostly non-orientable. It is very likely that this non-orientability is related to a non-zero winding number of the gauge field and the artificial configurations which we discussed in this article reflect properties of those field configuration which saturate the path integral. The discrepancies which we have indicated are not due to the coarseness of the lattice, the traces of all plaquettes are close to unity, $\operatorname{tr}\left(\mathbb{1}-U_{\mu \nu}\right) \leq 1-\cos \frac{\pi}{18}=0.015$ but probably due to singularities in the gauge field related to non-trivial mappings of Wilson lines.

\section{Acknowledgment}

This study was partially supported by the Austria Science Fund (FWF) under grant P20016-N16.

[1] G. 't Hooft, Nucl. Phys. B138, 1 (1978).

[2] P. Vinciarelli, Phys. Lett. B78, 485 (1978).

[3] T. Yoneya, Nucl. Phys. B144, 195 (1978).

[4] J. M. Cornwall, Nucl. Phys. B157, 392 (1979).

[5] G. Mack and V. B. Petkova, Ann. Phys. 123, 442 (1979).

[6] H. B. Nielsen and P. Olesen, Nucl. Phys. B160, 380 (1979).

[7] L. Del Debbio, M. Faber, J. Greensite, and S. Olejnik, Phys. Rev. D55, 2298 (1997), hep-lat/9610005.

[8] T. G. Kovacs and E. T. Tomboulis, Phys. Rev. D57, 4054 (1998), hep-lat/9711009.

[9] H. Reinhardt and M. Engelhardt, Center vortices in continuum yang-mills theory, in Quark Confinement and the Hadron Spectrum IV, edited by W. Lucha and K. M. Maung, pp. 150-162, World Scientific, 2002, hep-th/0010031.

[10] P. de Forcrand and M. D'Elia, Phys. Rev. Lett. 82, 4582 (1999), hep-lat/9901020.

[11] C. Alexandrou, P. de Forcrand, and M. D'Elia, Nucl. Phys. A663, 1031 (2000), hep-lat/9909005. 
[12] M. Engelhardt, Nucl. Phys. B638, 81 (2002), hep-lat/0204002.

[13] R. Narayanan and H. Neuberger, Nucl. Phys. B443, 305 (1995), hep-th/9411108.

[14] H. Neuberger, Phys. Lett. B417, 141 (1998), hep-lat/9707022.

[15] M. Luscher, Phys. Lett. B428, 342 (1998), hep-lat/9802011.

[16] M. F. Atiyah and I. M. Singer, Annals Math. 93, 139 (1971).

[17] A. S. Schwarz, Phys. Lett. B67, 172 (1977).

[18] L. S. Brown, R. D. Carlitz, and C.-k. Lee, Phys. Rev. D16, 417 (1977).

[19] D. H. Adams, J. Math. Phys. 42, 5522 (2001), hep-lat/0009026.

[20] R. G. Edwards, U. M. Heller, and R. Narayanan, Nucl. Phys. B540, 457 (1999), hep-lat/9807017.

[21] M. Luscher, Nucl. Phys. B549, 295 (1999), hep-lat/9811032.

[22] H. Neuberger, Phys. Rev. D61, 085015 (2000), hep-lat/9911004.

[23] P. Di Vecchia, K. Fabricius, G. C. Rossi, and G. Veneziano, Nucl. Phys. B192, 392 (1981).

[24] P. Di Vecchia, K. Fabricius, G. C. Rossi, and G. Veneziano, Phys. Lett. B108, 323 (1982).

[25] P. van Baal, Commun. Math. Phys. 85, 529 (1982).

[26] J. Greensite, Prog. Part. Nucl. Phys. 51, 1 (2003), hep-lat/0301023.

[27] H. Reinhardt, O. Schroeder, T. Tok, and V. C. Zhukovsky, Phys. Rev. D66, 085004 (2002), hep-th/0203012.

[28] R. Bertle, M. Faber, J. Greensite, and S. Olejnik, JHEP 03, 019 (1999), hep-lat/9903023.

[29] In this work, we denote as "zero-mode" any eigenmode of $D^{\dagger} D$ of the overlap Dirac operator (2) with eigenvalue smaller than $10^{-5}$. If such modes exist of both positive and negative chirality they are most likely not exact zero-modes, but lifted slightly above zero pairwise. This numerical inaccuracy does not affect the value of the index (1). 